\title{
Spanning Boundaries into Remote Communities: An Exploration of Experiences with Telehealth Chronic Disease Self-Management Programs in Rural Northern Ontario, Canada
}

Sara J.T. Guilcher, PhD, $P T^{1-3}$ Tarik Bereket, $M A_{1}^{1,3}$ Jennifer Voth, $M A, P h D^{4}$ Vinita A. Haroun, $M S c^{5}{ }^{5}$ and Susan B. Jaglal, $P h D^{1-4}$

${ }^{1}$ Women's College Research Institute, Toronto, Ontario, Canada.

${ }^{2}$ Centre for Research on Inner City Health, St. Michael's Hospital, Toronto, Ontario, Canada.

${ }^{3}$ Department of Physical Therapy, University of Toronto, Toronto, Ontario, Canada.

${ }^{4}$ Toronto Rehabilitation Institute-University Health Network, Toronto, Ontario, Canada.

${ }^{5}$ Public Health Ontario, Toronto, Ontario, Canada.

\begin{abstract}
Background: In rural and remote settings, providing education programs for chronic conditions can be challenging because of the limited access and availability of healthcare services. The purpose of this study was to explore the experiences of participants in a chronic disease self-management program via telehealth (tele-CDSMP) and to identify facilitators and barriers to inform future tele-CDSMP delivery models. Materials and Methods: Nineteen tele-CDSMP courses were delivered to 13 Northern Ontario (Canada) communities. Two types of group were delivered: (1) single telehealth site (one community formed a self-management group linked to program leaders via telehealth) and (2) multiple telehealth sites (several remote communities were linked to each other and program leaders via telehealth). Following the completion of the courses, participants were invited to partake in a focus group. Results: Overall, 44 people participated in the focus groups. Four main themes were identified by tele-CDSMP participants related to the overall experience of the program: (1) bridging the access gap, (2) importance of group dynamics, (3) importance of strong leaders, and (4) preference for extended session time. Key barriers were related to transportation, lack of session time, and access to Internet-based resources. The main facilitators were having strong program leaders, encouraging the development of group identity, and providing enough time to be comfortable with technology. Conclusions: Our findings suggest overall the tele-CDSMP was a positive experience for participants and that tele-CDSMPs are an effective option to increasing access to more geographically isolated communities.
\end{abstract}

Key words: telehealth, telemedicine, policy

\section{Introduction}

$\mathrm{n}$ Canada, the prevalence of persons living with a chronic health condition is approximately 9 million, and 75\% of seniors have one or more chronic conditions. ${ }^{1}$ Previous research has identified persons living in rural Canada as exhibiting less healthy behaviors (e.g., decreased physical activity and decreased consumption of fruits and vegetables) ${ }^{2}$ and having a higher risk of mortality with respect to living with chronic conditions (e.g., respiratory disease, circulatory disease, and diabetes). ${ }^{2-4}$

Providing education programs targeted toward effectively managing chronic conditions to people living in rural and remote settings is challenging, given the limited access and availability of healthcare and community-based services. ${ }^{2-4}$ Using telehealth to deliver these educational programs may be a cost-effective way of providing access to isolated communities. We recently delivered the Stanford Chronic Disease Self-Management Program via telehealth (tele-CDSMP) to rural communities in Northern Ontario. ${ }^{5}$ The Stanford Chronic Disease Self-Management Program ${ }^{6}$ is a 6 -week health education program that helps people develop effective self-management skills to cope with the physical and emotional challenges of living with a chronic disease. This program has demonstrated effectiveness in improving health-related attitudes, behaviors, and health status in a variety of patient populations across an array of geographical areas. ${ }^{7}$

In particular, we showed effectiveness of the tele-CDSMP in rural Northern Ontario, as improvements in self-efficacy, health status, and health behaviors were demonstrated. ${ }^{5}$ Statistically significant improvements from baseline to 4-month follow-up were found for self-efficacy, exercise behavior, cognitive symptom management, communication with physicians, role function, psychological well-being, energy, health distress, and self-rated health. Similarly, Lorig et al. ${ }^{8}$ reported favorable results supporting the Internet as an effective mode of delivery of the CDSMP, with improvements shown in health status, self-efficacy, and health behavior sustained at one year. However, less favorable results were found for telephone delivery. ${ }^{9}$ Jerant et al. ${ }^{9}$ conducted a randomized control trial comparing the effectiveness of an in-home delivery and telephone delivery compared with usual care. The in-home delivery led to improvements in self-efficacy at 6 weeks but no sustained effects at one year. The telephone delivery group had no significant effects on any health status or healthcare use outcomes. ${ }^{9}$

Currently, there is a gap in the literature with respect to understanding the experiences of participants in these different media for the delivery of the CDSMP. A recent meta-analysis on selfmanagement education programs highlighted the need to 
Table 1. Demographics of Participating Communities in Northern Ontario by Type of Telehealth Chronic Disease Self-Management Program Course Offered

\begin{tabular}{|c|c|c|c|c|}
\hline \multirow[b]{2}{*}{ COMMUNITY } & \multirow[b]{2}{*}{$\begin{array}{l}\text { POPULATION } \\
\text { IN } 2006\end{array}$} & \multirow{2}{*}{$\begin{array}{c}\text { MEDIAN } \\
\text { AGE } \\
\text { (YEARS) }\end{array}$} & \multicolumn{2}{|c|}{ TH GROUP } \\
\hline & & & $\begin{array}{l}\text { SINGLE- } \\
\text { SITE }\end{array}$ & $\begin{array}{l}\text { MULTIPLE- } \\
\text { SITE }\end{array}$ \\
\hline $\operatorname{lgnace}^{a}$ & 1,431 & 42.5 & $\sqrt{ }$ & - \\
\hline Terrace Bay & 1,625 & 45.6 & $\sqrt{ }$ & - \\
\hline Nipigon $^{a}$ & 1,752 & 43.6 & - & $\sqrt{ }$ \\
\hline Manitouwadge ${ }^{a}$ & 2,300 & 42.8 & $\sqrt{ }$ & - \\
\hline Atikokan & 3,293 & 43.0 & $\sqrt{ }$ & - \\
\hline Blind River $^{a}$ & 3,780 & 46.1 & - & $\sqrt{ }$ \\
\hline Red Lake & 4,526 & 37.9 & - & $\sqrt{ }$ \\
\hline Sioux Lookout & 5,183 & 35.1 & - & $\sqrt{ }$ \\
\hline Cochrane & 5,487 & 41.1 & - & $\sqrt{ }$ \\
\hline Sturgeon Falls ${ }^{a}$ & 6,383 & 44.7 & $\sqrt{ }$ & - \\
\hline Kirkland Lake ${ }^{a}$ & 8,248 & 43.8 & $\sqrt{ }$ & $\sqrt{ }$ \\
\hline Kenora & 15,177 & 42.4 & $\sqrt{ }$ & $\sqrt{ }$ \\
\hline Huntsville $^{a}$ & 18,280 & 43.4 & $\sqrt{ }$ & $\sqrt{ }$ \\
\hline Total $(n=13)$ & 77,465 & 42.46 & & \\
\hline
\end{tabular}

${ }^{a}$ Communities that participated in focus groups.

$\mathrm{TH}$, telehealth

qualitatively explore the participants' evaluation of the CDSMP and how different methods of delivery affect their overall experience. ${ }^{7}$ Thus, the purpose of this study was to explore the experiences of participants in tele-CDSMP and to identify facilitators and barriers to inform future tele-CDSMP delivery models.

\section{Materials and Methods}

\section{SETTING AND FOCUS GROUP RECRUITMENT}

Nineteen tele-CDSMP courses were delivered to 13 Northern Ontario communities with populations of less than 40,000 (Table 1 gives community demographics) with existing telehealth infrastructure between September 2007 and June 2008. ${ }^{5}$ The tele-CDSMP was adapted for telehealth delivery from the previously evaluated CDSMP developed by Stanford University. ${ }^{6,10,11}$

The telehealth technology used a set-top Tandberg 880 (Tandberg, Oslo, Norway) videoconferencing system with a bandwidth of 512 kilobits per second. Using this existing telehealth infrastructure allowed for two possible types of group delivery of the tele-CDSMP: (1) telehealth single-site groups, where participants from one community form a selfmanagement group linked to program leaders via telehealth, and (2) telehealth multiple-site groups, in which participants from several remote communities are linked to each other and program leaders via telehealth to form a self-management group (Fig. 1). A minimum of 10 people was enrolled for a single-site course, and efforts were made to recruit at least two participants from each community in a multiple-site course.

Following the completion of the courses, all participants were informed about optional focus groups and asked whether they were interested in providing feedback on experiences with the tele-CDSMP. Interested participants from selected communities were then provided with information and consent packages inviting them to participate in a one-time focus group. Efforts were made to achieve maximal variation on community size, as well as type of telehealth delivery (multiple-site or single-site; Table 1), and a minimum of six participants for each focus group. ${ }^{12}$ Ignace, Nipigon, Manitouwadge, Blind River, Sturgeon Falls, Kirkland Lake, and Huntsville were the Northern Ontario communities $(n=7)$ that participated in focus groups.

\section{TELE-CDSMP PARTICIPANTS AND FOCUS GROUP MEMBERS}

Eligible tele-CDSMP participants had a self-reported physician diagnosis of chronic lung disease, heart disease, stroke, or arthritis. Participants were 45 years of age or older and required to be able to speak and read English and be able to attend a 2.5-h session, once a week, for a total of 6 weeks enrolled in one of the 19 courses offered (female subjects, $n=161$ [75.6\%]; male subjects, 52 [24.4\%]). Of the enrolled participants, 92 people were interested in being part of the focus group activities, and 44 were selected and participated (female subjects, 30 [68\%]; male subjects, 14 [32\%]). This study was approved by the Research Ethics Board at Women's College Hospital.

\section{FOCUS GROUP DATA COLLECTION AND ANALYSIS}

The focus group questions (Table 2) were aimed at exploring the overall experiences of participants in the tele-CDSMP and to gain a greater understanding of the facilitators and barriers of telehealth delivery. The focus groups were approximately 60-90 min in duration. Two members of the research team facilitated the focus group activity via telehealth, where one member asked the main questions, and the other took detailed notes and offered useful prompts for particular responses that needed further elaboration.

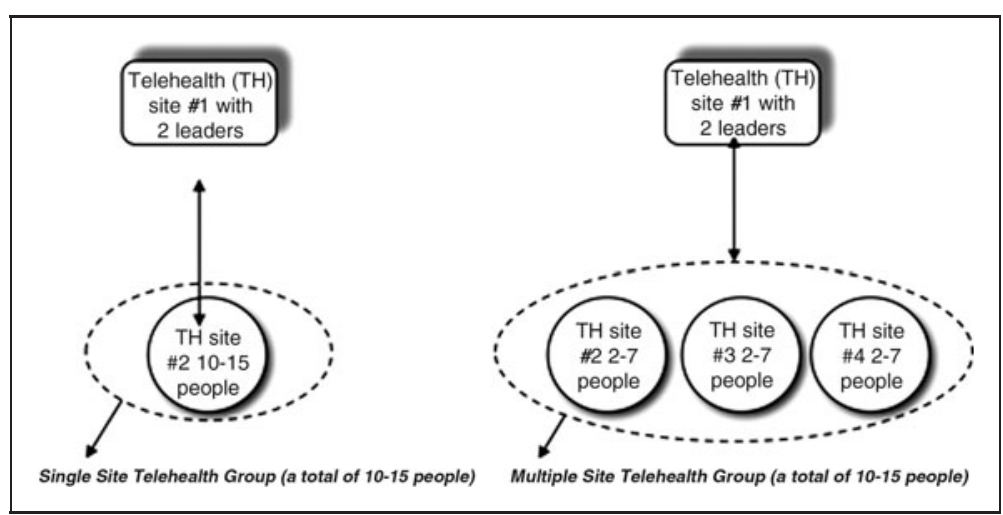

Fig. 1. Single-site versus multiple-site chronic disease self-management programs via telehealth. 


\section{GUILCHER ET AL.}

Table 2. Focus Group Questions for Program Participants

1. Can you tell us about your experience with the telehealth chronic disease self-management program?

1.1. Can you describe what worked best for you in the use of telehealth? Can you give examples?

1.2. Can you describe what didn't work well for you about the use of telehealth? Can you give examples?

1.3. What improvements would you suggest to resolve this issue?

2.1. The program leaders play a critical role in facilitating the program. Can you tell us about your experience with your leaders?

2.2. If applicable, in what ways if any did having a replacement leader influence how the program was facilitated? Did this influence your experience in the program?

3. Do you have any feedback regarding the materials used during the course (e.g., handouts)?

4.1. Do you have any overall suggestions as to how we can improve the program?

4.2. Do you have any other thoughts or comments about the program or about the use of telehealth that you would like to add?

All qualitative discussions were digitally recorded and transcribed verbatim. All identifiers were systematically removed from transcripts. Following each focus group session, reflective notes were also documented and reviewed. All transcripts were entered into NVivo version 7.0, an advanced storage-code-and-retrieval software program (www .qsrinternational.com). The data were coded electronically according to standard qualitative coding techniques. ${ }^{13}$ The data analysis began with an inductive descriptive process of sorting and defining the data. ${ }^{14}$ This process entailed dividing the text of the transcripts into segments of text and attaching descriptive codes of analysis to these segments. ${ }^{14}$ The descriptive codes were then grouped into broad topicoriented categories, and all text segments belonging to the same category are compared. ${ }^{14}$ Ultimately, the topic-oriented categories were refined and formulated into fewer analytical categories through an inductive, iterative process of reading through the categorized quotes from participants. Data analysis was conducted concurrently with data collection, a process referred to as interim analysis, ${ }^{14}$ which allows for confirmation, modification, or rejection of emerging themes as new transcripts were analyzed. ${ }^{14,15}$ To ensure that the data were being coded in a consistent manner, several members of the research team independently coded transcripts, and analyses were compared. ${ }^{14,15}$

\section{Results}

Four main themes identified by tele-CDSMP participants related to the overall experience of the program: (1) bridging the access gap, (2) importance of group dynamics, (3) importance of strong leaders, and (4) preference for extended session time. Furthermore, several barriers and facilitators were identified to help inform future implementation considerations (Table 3).

\section{BRIDGING THE ACCESS GAP}

Program participants expressed a preference for in-person classes due to some challenges with the telehealth technology such as intermittent audio and visual problems. However, despite this preference, participants noted that the tele-CDSMP is a welcomed alternative to bridge the gap in healthcare provision to rural communities (FG indicates the focus group):
We're also all here because we thought that this program would be beneficial to our community. We don't want to see it not come back again...I've always said in southern Ontario, you have much more access to seminars, courses than you do here and we are very anxious to learn all we can. (FG 6, female participant)

There was an acknowledgment among participants that because of various physical challenges (e.g., transportation) and system challenges (e.g., availability of services) that are inherent with the remote settings, delivering a program in-person was likely not a feasible option:

I think it's good to really get help faster and not have to travel great distances to get the help. That's what I find best about telehealth. Sometimes you can avoid problems by getting to them sooner. I think telehealth in general is a great thing. It's a real advantage. (FG 4, male participant)

\section{IMPORTANCE OF GROUP DYNAMICS}

In both the single-site and multiple-site groups, participants noted that group formation occurred despite some of the challenges with the technology. Participants greatly valued the presence of group members, and participants noted the success of the course seemed to be strongly influenced by the positive group dynamics. Participants identified four key group functions: (a) sharing a common experience, (b) building social connections, (c) information-sharing strategies, and (d) developing accountability and motivation via peer coaching.

Sharing a common experience. Participants commented that the group dynamic provided a mechanism for sharing feelings and experiences related to the chronic condition(s). Participants noted that there could be stigmatization in talking about the chronic condition with individuals in their everyday life and that the tele-CDSMP group provided a safe and open forum to share experiences:

I found it very easy to be expressive. In fact, I really opened up...the first time I really opened up was here. Like my wife heard me say things that I had never even talked to her about. (FG 7, male participant) 
Table 3. Main Barriers and Facilitators for Future Telehealth Program Implementation

BARRIERS

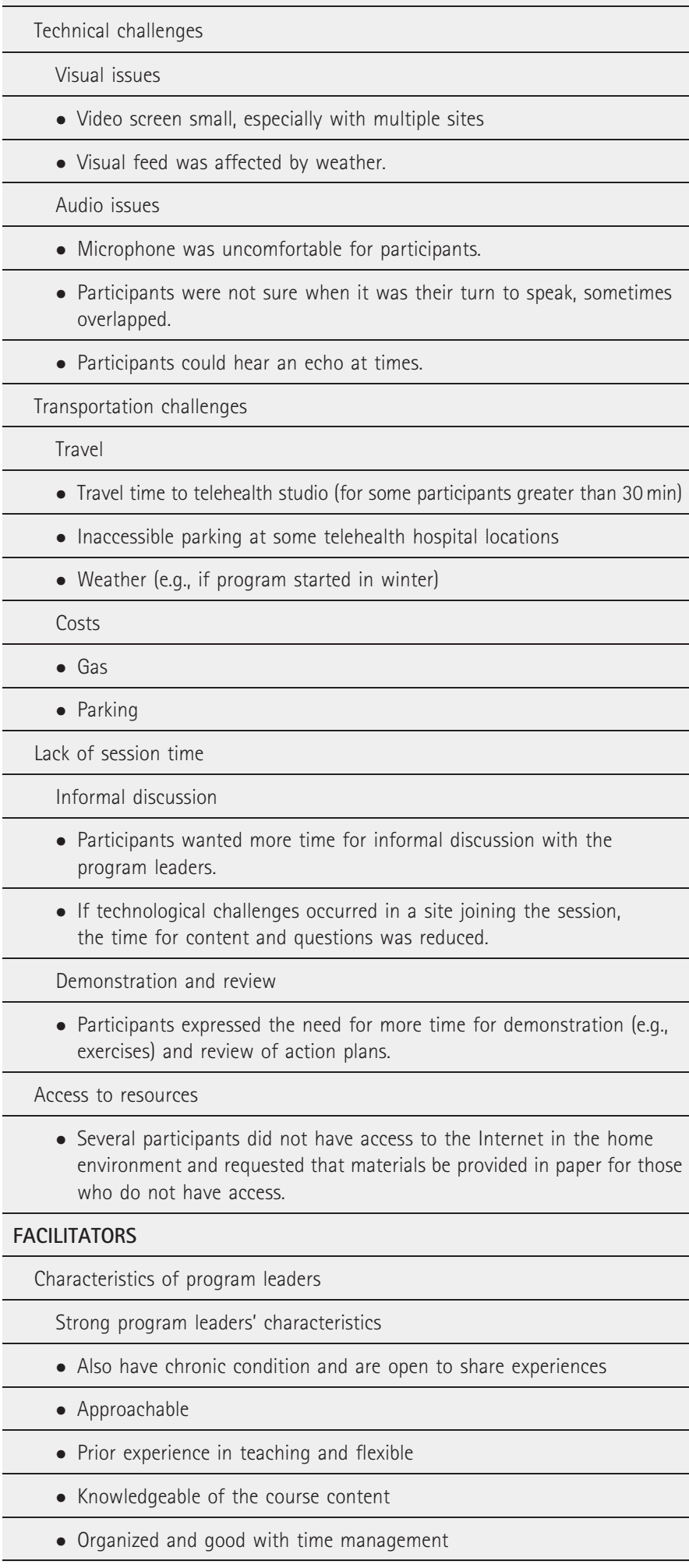

\section{Table 3. continued}

- Demonstrate exemplary action planning

- Good facilitator of group discussions, flexible

Encouraging the development of group identity

- Strong group dynamics

- Informal interactions within the program and outside the program

- An informal meet and greet prior to the official program starting

- Small groups, especially if multiple site, to allow opportunity for participants to share thoughts

\author{
Time \\ - Participants developed a sense of familiarity with the technology \\ as time progressed
}

Building social connections. Participants noted that program helped encourage new friendships and minimized social isolation, which are especially important for individuals living on their own. Many participants voiced that they have been staying in contact with each other after the tele-CDSMP and that these bonds have been a motivating factor in their attempts to stay on track with goals set at the onset of the program:

...when you run into somebody that was in the group and they know what your problems are and you know what their problems are, it makes it more personal. You could say "well how is your back today" or "how are you doing on your exercise program" or put some input into their help instead of just saying "it's a nice day today" [...] it's more personal. I think that came from this program. (FG 1, female participant)

The single-site groups seemed to have established stronger bonding social capital, reflecting closer and more intimate connections, compared with the multiple-site group. Participants commented that the opportunity to have informal interactions in the local community was helpful in fostering continued friendships:

I don't have anybody at home to discuss with or share with. For me, I really enjoyed the sessions. They were not only enlightening. There are other people like that. It was a good social outlet for me too and I enjoyed that. (FG 7, female participant)

In theory, the multiple-site groups should foster information sharing across numerous communities, with a potential to enhance participants bridging social capital. However, participants noted more challenges with the multiple-site group in connecting with individuals both within and outside the program (discussed in more detail under the barriers section).

Information-sharing strategies. The group environment facilitated information exchange regarding existing community resources and individual strategies used on a day-to-day basis that encourage healthy 


\section{GUILCHER ET AL.}

living (e.g., nutrition, exercises). Participants in the multiple-site groups acknowledged that being linked to different communities increased the opportunity to access information from different communities:

I passed on quite a few hints.... While I sat there reading my book or waiting for someone to come to my table, I would do leg exercises underneath the table and hope people who are standing there wouldn't think I was too nuts or something. Like folding your clothes, I put the clothes in front on the couch and I stand behind the couch so I have to bend over the couch but not lean on it to pick up the clothes and fold them, just little hints like that I passed on to the group. If it works, great. (FG 3, female participant)

Developing accountability and motivation via peer coaching. Participants noted that individuals helped coach each other by providing encouragement and accountability for the action plans. Particularly with the single-site groups, the support extended beyond the tele-CDSMP meeting times and continued outside the telehealth studio to ensure that practical guidelines are translated into performed action plans. Participants noted that the exchanges during the program were an important factor in strengthening the commitment that they had for the completion of the 6-week program:

I do exercises 5 days a week at least. I walk in the morning 1 to 2 kilometers and work out on 3 or 4 different machines each day, 15 minutes or 20 minutes. I'm 86, going on 87 . I feel a lot better for it....I've lost 5 pounds. I'm on a point system. The three of us that walk together, we're all on a point system. We meet each day to write it down and count up our points. (FG 1, female participant)

\section{STRONG LEADERS WERE ESSENTIAL TO OVERCOME CHALLENGES WITH TECHNOLOGY}

Participants noted that it was important for leaders to be knowledgeable in the subject matter, to be organized, and to provide flexibility to group needs:

I think because of the three sites, it made them very rigid so there was less flexibility in terms of what they could do...it's one thing to have a plan but you sometimes have to deviate from that and you can't if you're on a rigid schedule. That's the problem...(FG 6, female participant)

Participants were able to recognize when CDSMP leaders were well prepared and allowed some flexibility in the discussions, such that participants could explore topics of interest, but also ensure that all subject matter was thoroughly covered. Participants noted that it was important for leaders to be strong facilitators of group discussions, especially for the multiple-site groups. For example, participants indicated they were hesitant at times to speak into the microphone and that effective facilitation was important to encourage participation:

People have a lot to say and they have things on their mind that they won't just speak out into the TV for everybody to hear. (FG 5, female participant)

\section{PREFERENCE FOR EXTENDED SESSION TIME}

Participants noted that they would have preferred longer individual sessions or a longer overall course to review material. For example, participants described not having enough time to review action plans or an opportunity for informal question and answers with the facilitators. The lack of time seemed particularly noted with the multiple-site groups, as participants described delays in starting due to different sites joining the session. In a face-to-face session, there was opportunity for informal discussions with facilitators, but the telehealth delivery did not provide the same access to facilitators:

If you're in a person to person situation, somebody is teaching you here in the same room, if you weren't comfortable enough asking a question in front of the group, you could always discuss it with an individual afterwards to say, "I was wondering about A, B, or C" whereas in this situation, you don't have that option. (FG 6, female participant)

Additionally, participants described that while group dynamics formed, it took a few sessions before participants felt comfortable with the technology format:

...that made it more interesting to have another group in another place to talk about different things. I found that very good. When I first started it, the first week or so, the teleconferencing I didn't like it that well. But the more you got into it the better it got. The first few weeks, I told my wife I didn't want to go anymore. But after a few weeks, it got a little better...(FG 6, male participant)

\section{SUMMARY OF BARRIERS AND FACILITATORS}

The main barriers and facilitators described by participants are shown in Table 3. Participants highlighted the following as the main barriers that provided challenges with the telehealth technology: transportation, lack of session time, and access to Internet-based resources. The main facilitators that participants identified as key contributors to a successful program were having strong program leaders, encouraging the development of group identity, and providing enough time to be comfortable with the initially foreign technology.

\section{Discussion}

Effectively managing chronic conditions is a major focus of current health policy in which self-management is increasingly seen as a component. ${ }^{16}$ Given this, it is important that we understand how different and innovative delivery methods, such as telehealth, contribute toward the effective delivery to isolated communities. Our findings provide important insights into the overall experiences of the telehealth program and the components that facilitate or hinder implementation. We identified the following components that could make the delivery of the tele-CDSMP more effective in rural communities: (1) bridging the access gap, (2) importance of group dynamics, (3) importance of strong leaders, and (4) preference for extended session time.

The group formation, in particular, was identified as a critical component for a successful program. Similar to other studies that have explored relationships within groups, ${ }^{17,18}$ our findings suggest 
the strong formation of group identity and cohesion facilitated the overall positive experience of the program. The group dynamic could be a critical component to the success of the CDSMP. Jerant et al. ${ }^{9}$ did not report significant sustained effects with the in-home or telephone delivery mode, whereas Lorig et al. ${ }^{8}$ in an Internet-based study and Jaglal et al. ${ }^{5}$ showed positive changes in self-efficacy, health behaviors, and health status. The latter two studies had components of peer interactions and social networking. Based on our qualitative results, building strong group dynamics was essential to positive experiences with the program. However, based on our focus groups, there seemed to be more challenges in establishing a strong group identity with the multiple-site courses. Technological challenges such as delayed start times and the lack of informal interactions both within and outside the program for the multiple-site courses seemed to be the biggest factors impacting group cohesion. Yet, health outcomes between the single and multiple-site groups were similar. ${ }^{5}$

Our findings suggest overall the tele-CDSMP was a positive experience for all participants. However, participants seemed to prefer the single-site course compared with the multiple-site methods. Most participants initially expressed some reservations about the use of telehealth; however, over time participants became more comfortable with the delivery method. In the future, tele-CDSMP programs should provide opportunities for participants to have more time to have questions answered by facilitators and for multiple-site groups to have slightly longer sessions to accommodate any technical challenges.

In summary, tele-CDSMP is a practical way for reaching people in rural communities who otherwise may not have access to such programs. Given the prevalence of chronic conditions in rural communities, our findings suggest that tele-CDSMPs are an effective option to increasing access to more geographically isolated communities.

\section{Acknowledgments}

We would like to acknowledge the funding support provided by the Canadian Institutes of Health Research (CIHR) (funding reference number MOP 81099) and our partnership with the Ontario Telemedicine Network and its member sites. S.B.J. is the Toronto Rehabilitation Institute Chair at the University of Toronto. S.J.T.G. is a CIHR Strategic Training Fellow in the ACHIEVE Research Partnership: Action for Health Equity Interventions [From the] Centre for Research on Inner City Health, the Keenan Research Centre in the Li Ka Shing Knowledge Institute of St. Michael's Hospital.

\section{Disclosure Statement}

No competing financial interests exist.

\section{REFERENCES}

1. Broemeling AM, Watson DE, Prebtani F. Population patterns of chronic health conditions, co-morbidity and healthcare use in Canada: Implications for policy and practice. Healthc 0 2008;11:70-76.

2. Canadian Institute for Health Information. How healthy are rural Canadians? An assessment of their health status and health determinants. Ottawa: Canadian Population Health Initiative, 2006.
3. Chan B, Young W. Chapter 1: Burden of cardiac disease. In: Naylor D, Slaughter $P$, eds. ICES research atlas: Cardiovascular health and services in Ontario. Toronto: Institute for Clinical Evaluative Studies, 1999. Available at: http:// www.ices.on.ca/file/3_cva_chapter1.pdf (last accessed October 1, 2013).

4. Perruccio AV, Badley EM, Guan J. Chapter 2: Burden of disease. In: Williams J, Badley $\mathrm{E}_{1}$ eds. Arthritis and related conditions in Ontario: An ICES practice atlas. Toronto: Institute for Clinical Evaluative Studies, 2004:15-40. Available at: http://www.acreu.ca/pdf/ICES_atlas-intro.pdf (last accessed October 1, 2013).

5. Jaglal SB, Haroun VA, Salbach NM, et al. Increasing access to chronic disease self-management programs in rural and remote communities using telehealth. Telemed J E Health 2013;19:467-473.

6. Lorig KR, Sobel DS, Stewart AL, et al. Evidence suggesting that a chronic disease self-management program can improve health status while reducing hospitalization: A randomized trial. Med Care 1999;37:5-14.

7. Centers for Disease Control and Prevention. Sorting through the evidence for the arthritis self-management program and the chronic disease selfmanagement program: Executive summary of ASMP/CDSMP meta-analyses. Atlanta: Centers for Disease Control and Prevention, 2011.

8. Lorig KR, Ritter PL, Laurent DD, Plant K. Internet-based chronic disease selfmanagement: A randomized trial. Med Care 2006;44:964-971.

9. Jerant A, Moore-Hill M, Franks P. Home-based, peer-led chronic illness selfmanagement training: Findings from a 1-year randomized controlled trial. Ann Fam Med 2009;7:319-327.

10. Lorig KR, Sobel DS, Ritter PL, Laurent $D$, Hobbs M. Effect of a self-management program on patients with chronic disease. Eff Clin Pract 2001;4:256-262.

11. Lorig KR, Holman H. Self-management education: History, definition, outcomes, and mechanisms. Ann Behav Med 2003;26:1-7.

12. Krueger RA, Casey MA. Focus groups: A practical guide for applied research. Los Angeles: Sage Publications, 2009.

13. Denzin NK, Lincoln YS. Collecting and interpreting qualitative materials. Thousand Oaks, CA: Sage Publications, 1998.

14. Miles M, Huberman A. Qualitative data analysis: An expanded sourcebook. Thousand Oaks, CA: Sage Publications, 1994.

15. Pope C, Ziebland S, Mays N. Qualitative research in health care. Analysing qualitative data. BMJ 2000;320:114-116.

16. Kennedy A, Rogers A, Bower P. Support for self care for patients with chronic disease. BMJ 2007;335:968-970.

17. Cameron JE. Social identity and the pursuit of possible selves: Implications for the psychological well-being of university students. Group Dynamics 1999;3:179-189.

18. Marmarosh C, Holtz A, Schottenbauer M. Group cohesiveness, group-derived collective self-esteem, group-derived hope, and the well-being of group therapy members. Group Dynamics 2005;9:32-44.

Address correspondence to:

Susan B. Jaglal, PhD

Department of Physical Therapy

Toronto Rehabilitation Institute

University of Toronto

160-500 University Avenue

Toronto, ON M5G $1 \mathrm{~V}$

Canada

E-mail: susan.jaglal@utoronto.ca

Received: February 27, 2013

Revised: April 12, 2013

Accepted: April 12, 2013 